\title{
Existence of solutions for a coupled system of fractional differential equations at resonance
}

\author{
Zhigang Hu, Wenbin Liu* and Taiyong Chen
}

\section{"Correspondence:} wblium@163.com

Department of Mathematics, China University of Mining and Technology, Xuzhou, 221008

People's Republic of China

\begin{abstract}
In this paper, by using the coincidence degree theory, we study the existence of solutions for a coupled system of fractional differential equations at resonance. A new result on the existence of solutions for a fractional boundary value problem is obtained.

MSC: $34 \mathrm{~B} 15$

Keywords: fractional differential equation; boundary value problem; coincidence degree theory; resonance
\end{abstract}

\section{Introduction}

In recent years, the fractional differential equations have received more and more attention. The fractional derivative has been occurring in many physical applications such as a non-Markovian diffusion process with memory [1], charge transport in amorphous semiconductors [2], propagations of mechanical waves in viscoelastic media [3], etc. Phenomena in electromagnetics, acoustics, viscoelasticity, electrochemistry and material science are also described by differential equations of fractional order (see [4-9]).

Recently, boundary value problems for fractional differential equations have been studied in many papers (see [10-25]). Moreover, the existence of solutions to a coupled systems of fractional differential equations have been studied by many authors (see [26-33]). But the existence of solutions for a coupled system of fractional differential equations at resonance are seldom considered. Motivated by all the works above, in this paper, we consider the following boundary value problem (BVP for short) for a coupled system of fractional differential equations given by

$$
\left\{\begin{array}{l}
D_{0^{+}}^{\alpha} u(t)=f\left(t, v(t), v^{\prime}(t)\right), \quad t \in(0,1), \\
D_{0^{+}}^{\beta} v(t)=g\left(t, u(t), u^{\prime}(t)\right), \quad t \in(0,1), \\
u(0)=v(0)=0, \quad u^{\prime}(0)=u^{\prime}(1), \quad v^{\prime}(0)=v^{\prime}(1),
\end{array}\right.
$$

where $D_{0^{+}}^{\alpha}, D_{0^{+}}^{\beta}$ are the standard Caputo fractional derivatives, $1<\alpha \leq 2,1<\beta \leq 2$ and $f, g:[0,1] \times \mathbb{R}^{2} \rightarrow \mathbb{R}$ is continuous.

The rest of this paper is organized as follows. Section 2 contains some necessary notations, definitions and lemmas. In Section 3, we establish a theorem on the existence of solutions for BVP (1.1) under nonlinear growth restriction of $f$ and $g$, basing on the coin-

(c) 2012 Hu et al.: licensee Springer. This is an Open Access article distributed under the terms of the Creative Commons Attribution License (http://creativecommons.org/licenses/by/2.0), which permits unrestricted use, distribution, and reproduction in any medium, provided the original work is properly cited. 
cidence degree theory due to Mawhin (see [34]). Finally, in Section 4, an example is given to illustrate the main result.

\section{Preliminaries}

In this section, we will introduce some notations, definitions and preliminary facts which are used throughout this paper.

Let $X$ and $Y$ be real Banach spaces, and let $L: \operatorname{dom} L \subset X \rightarrow Y$ be a Fredholm operator with index zero, and $P: X \rightarrow X, Q: Y \rightarrow Y$ be projectors such that

$$
\begin{aligned}
& \operatorname{Im} P=\operatorname{Ker} L, \quad \operatorname{Ker} Q=\operatorname{Im} L, \\
& X=\operatorname{Ker} L \oplus \operatorname{Ker} P, \quad Y=\operatorname{Im} L \oplus \operatorname{Im} Q .
\end{aligned}
$$

It follows that

$$
\left.L\right|_{\operatorname{dom} L \cap \operatorname{Ker} P}: \operatorname{dom} L \cap \operatorname{Ker} P \rightarrow \operatorname{Im} L
$$

is invertible. We denote the inverse by $K_{P}$.

If $\Omega$ is an open bounded subset of $X$, and $\operatorname{dom} L \cap \bar{\Omega} \neq \varnothing$, the map $N: X \rightarrow Y$ will be called $L$-compact on $\bar{\Omega}$ if $Q N(\bar{\Omega})$ is bounded and $K_{P}(I-Q) N: \bar{\Omega} \rightarrow X$ is compact, where $I$ is an identity operator.

Lemma 2.1 [27] Let $L: \operatorname{dom} L \subset X \rightarrow Y$ be a Fredholm operator of index zero and $N: X \rightarrow$ $Y$ L-compact on $\bar{\Omega}$. Assume that the following conditions are satisfied:

(1) $L x \neq \lambda N x$ for every $(x, \lambda) \in[(\operatorname{dom} L \backslash \operatorname{Ker} L)] \cap \partial \Omega \times(0,1)$;

(2) $N x \notin \operatorname{Im} L$ for every $x \in \operatorname{Ker} L \cap \partial \Omega$;

(3) $\operatorname{deg}\left(\left.Q N\right|_{\operatorname{Ker} L}, \operatorname{Ker} L \cap \Omega, 0\right) \neq 0$, where $Q: Y \rightarrow Y$ is a projection such that $\operatorname{Im} L=\operatorname{Ker} Q$.

Then the equation $L x=N x$ has at least one solution in $\operatorname{dom} L \cap \bar{\Omega}$.

Definition 2.1 The Riemann-Liouville fractional integral operator of order $\alpha>0$ of a function $x$ is given by

$$
I_{0^{+}}^{\alpha} x(t)=\frac{1}{\Gamma(\alpha)} \int_{0}^{t}(t-s)^{\alpha-1} x(s) d s
$$

provided that the right-hand side integral is pointwise defined on $(0,+\infty)$.

Definition 2.2 The Riemann-Liouville fractional derivative of order $\alpha>0$ of a function $x$ is given by

$$
{ }^{R} D_{0^{+}}^{\alpha} x(t)=\frac{d^{n}}{d t^{n}} I_{0^{+}}^{n-\alpha} x(t)=\frac{1}{\Gamma(n-\alpha)} \frac{d^{n}}{d t^{n}} \int_{0}^{t}(t-s)^{n-\alpha-1} x(s) d s,
$$

where $n$ is the smallest integer greater than or equal to $\alpha$, provided that the right-hand side integral is pointwise defined on $(0,+\infty)$. 
Definition 2.3 The Caputo fractional derivative of order $\alpha>0$ of a function $x$ is given by

$$
D_{0^{+}}^{\alpha} x(t)={ }^{R} D_{0^{+}}^{\alpha}\left[x(t)-\sum_{k=0}^{n-1} \frac{x^{(k)}(0)}{k !} t^{k}\right],
$$

where $n$ is the smallest integer greater than or equal to $\alpha$, provided that the right-hand side integral is pointwise defined on $(0,+\infty)$.

Lemma 2.2 [35] Assume that $x \in C(0,1) \cap L(0,1)$ with a Caputo fractional derivative of order $\alpha>0$ that belongs to $C(0,1) \cap L(0,1)$. Then

$$
I_{0^{+}}^{\alpha} D_{0^{+}}^{\alpha} x(t)=x(t)+c_{0}+c_{1} t+c_{2} t^{2}+\cdots+c_{n-1} t^{n-1},
$$

where $c_{i} \in \mathbb{R}, i=0,1,2, \ldots, n-1$, here $n$ is the smallest integer greater than or equal to $\alpha$.

Lemma 2.3 [35] Assume that $\alpha>0$ and $x \in C[0,1]$. Then

$$
D_{0^{+}}^{\alpha} I_{0^{+}}^{\alpha} x(t)=x(t)
$$

In this paper, we denote $X=C^{1}[0,1]$ with the norm $\|x\|_{X}=\max \left\{\|x\|_{\infty},\left\|x^{\prime}\right\|_{\infty}\right\}$ and $Y=$ $C[0,1]$ with the norm $\|y\|_{Y}=\|y\|_{\infty}$, where $\|x\|_{\infty}=\max _{t \in[0,1]}|x(t)|$. Then we denote $\bar{X}=$ $X \times X$ with the norm $\|(u, v)\|_{\bar{X}}=\max \left\{\|u\|_{X},\|v\|_{X}\right\}$ and $\bar{Y}=Y \times Y$ with the norm $\|(x, y)\|_{\bar{Y}}=$ $\max \left\{\|x\|_{Y},\|y\|_{Y}\right\}$. Obviously, both $\bar{X}$ and $\bar{Y}$ are Banach spaces.

Define the operator $L_{1}: \operatorname{dom} L \subset X \rightarrow Y$ by

$$
L_{1} u=D_{0^{+}}^{\alpha} u,
$$

where

$$
\operatorname{dom} L_{1}=\left\{u \in X \mid D_{0^{+}}^{\alpha} u(t) \in Y, u(0)=0, u^{\prime}(0)=u^{\prime}(1)\right\} .
$$

Define the operator $L_{2}: \operatorname{dom} L_{2} \subset X \rightarrow Y$ by

$$
L_{2} v=D_{0^{+}}^{\beta} v,
$$

where

$$
\operatorname{dom} L_{2}=\left\{v \in X \mid D_{0^{+}}^{\beta} v(t) \in Y, v(0)=0, v^{\prime}(0)=v^{\prime}(1)\right\} .
$$

Define the operator $L: \operatorname{dom} L \subset \bar{X} \rightarrow \bar{Y}$ by

$$
L(u, v)=\left(L_{1} u, L_{2} v\right),
$$

where

$$
\operatorname{dom} L=\left\{(u, v) \in \bar{X} \mid u \in \operatorname{dom} L_{1}, v \in \operatorname{dom} L_{2}\right\} .
$$


Let $N: \bar{X} \rightarrow \bar{Y}$ be the Nemytski operator

$$
N(u, v)=\left(N_{1} v, N_{2} u\right)
$$

where $N_{1}: Y \rightarrow X$

$$
N_{1} v(t)=f\left(t, v(t), v^{\prime}(t)\right)
$$

and $N_{2}: Y \rightarrow X$

$$
N_{2} u(t)=g\left(t, u(t), u^{\prime}(t)\right)
$$

Then BVP (1.1) is equivalent to the operator equation

$$
L(u, v)=N(u, v), \quad(u, v) \in \operatorname{dom} L .
$$

\section{Main result}

In this section, a theorem on the existence of solutions for BVP (1.1) will be given.

Theorem 3.1 Let $f, g:[0,1] \times \mathbb{R}^{2} \rightarrow \mathbb{R}$ be continuous. Assume that

$\left(H_{1}\right)$ there exist nonnegative functions $p_{i}, q_{i}, r_{i} \in C[0,1](i=1,2)$ with

$$
\frac{\Gamma(\alpha) \Gamma(\beta)-4\left(Q_{1}+R_{1}\right)\left(Q_{2}+R_{2}\right)}{\Gamma(\alpha) \Gamma(\beta)}>0
$$

such that for all $(u, v) \in \mathbb{R}^{2}, t \in[0,1]$

$$
|f(t, u, v)| \leq p_{1}(t)+q_{1}(t)|u|+r_{1}(t)|v|,
$$

and

$$
|g(t, u, v)| \leq p_{2}(t)+q_{2}(t)|u|+r_{2}(t)|v|,
$$

where $P_{i}=\left\|p_{i}\right\|_{\infty}, Q_{i}=\left\|q_{i}\right\|_{\infty}, R_{i}=\left\|r_{i}\right\|_{\infty}(i=1,2)$;

$\left(H_{2}\right)$ there exists a constant $B>0$ such that for $\forall t \in[0,1],|u|>B, v \in \mathbb{R}$ either

$$
u f(t, u, v)>0, \quad u g(t, u, v)>0,
$$

or

$$
u f(t, u, v)<0, \quad u g(t, u, v)<0 ;
$$

$\left(H_{3}\right)$ there exists a constant $D>0$ such that for every $c_{1}, c_{2} \in \mathbb{R}$ satisfying $\min \left\{c_{1}, c_{2}\right\}>D$ either

$$
c_{1} N_{1}\left(c_{2} t\right)>0, \quad c_{2} N_{2}\left(c_{1} t\right)>0,
$$


or

$c_{1} N_{1}\left(c_{2} t\right)<0, \quad c_{2} N_{2}\left(c_{1} t\right)<0$.

Then BVP (1.1) has at least one solution.

Now, we begin with some lemmas below.

Lemma 3.1 Let $L$ be defined by (2.1), then

$$
\begin{aligned}
\operatorname{Ker} L & =\left(\operatorname{Ker} L_{1}, \operatorname{Ker} L_{2}\right)=\left\{(u, v) \in \bar{X} \mid(u, v)=\left(c_{1} t, c_{2} t\right), c_{1}, c_{2} \in \mathbb{R}\right\}, \\
\operatorname{Im} L & =\left(\operatorname{Im} L_{1}, \operatorname{Im} L_{2}\right) \\
& =\left\{(x, y) \in \bar{Y} \mid \int_{0}^{1}(1-s)^{\alpha-2} x(s) d s=0, \int_{0}^{1}(1-s)^{\beta-2} y(s) d s=0\right\} .
\end{aligned}
$$

Proof By Lemma 2.2, $L_{1} u=D_{0^{+}}^{\alpha} u(t)=0$ has the solution

$$
u(t)=c_{0}+c_{1} t, \quad c_{0}, c_{1} \in \mathbb{R} .
$$

Combining it with the boundary value conditions of BVP (1.1), one has

$\operatorname{Ker} L_{1}=\left\{u \in X \mid u=c_{1} t, c_{1} \in \mathbb{R}\right\}$.

For $x \in \operatorname{Im} L_{1}$, there exists $u \in \operatorname{dom} L_{1}$ such that $x=L_{1} u \in Y$. By Lemma 2.2, we have

$$
u(t)=\frac{1}{\Gamma(\alpha)} \int_{0}^{t}(t-s)^{\alpha-1} x(s) d s+c_{0}+c_{1} t
$$

Then, we have

$$
u^{\prime}(t)=\frac{1}{\Gamma(\alpha-1)} \int_{0}^{t}(t-s)^{\alpha-2} x(s) d s+c_{1} .
$$

By the conditions of BVP (1.1), we can get that $x$ satisfies

$$
\int_{0}^{1}(1-s)^{\alpha-2} x(s) d s=0 .
$$

On the other hand, suppose $x \in Y$ and satisfies $\int_{0}^{1}(1-s)^{\alpha-2} x(s) d s=0$. Let $u(t)=I_{0^{+}}^{\alpha} x(t)$, then $u \in \operatorname{dom} L_{1}$. By Lemma 2.3, we have $D_{0^{+}}^{\alpha} u(t)=x(t)$ so that $x \in \operatorname{Im} L_{1}$. Then we have

$$
\operatorname{Im} L_{1}=\left\{x \in Y \mid \int_{0}^{1}(1-s)^{\alpha-2} x(s) d s=0\right\} .
$$

Similarly, we can get

$$
\operatorname{Ker} L_{2}=\left\{v \in X \mid v=c_{2} t, c_{2} t \in \mathbb{R}\right\},
$$




$$
\operatorname{Im} L_{2}=\left\{y \in Y \mid \int_{0}^{1}(1-s)^{\beta-2} y(s) d s=0\right\}
$$

Then, the proof is complete.

Lemma 3.2 Let $L$ be defined by (2.1), then $L$ is a Fredholm operator of index zero, and the linear continuous projector operators $P: \bar{X} \rightarrow \bar{X}$ and $Q: \bar{Y} \rightarrow \bar{Y}$ can be defined as

$$
\begin{aligned}
& P(u, v)=\left(P_{1} u, P_{2} v\right)=\left(u^{\prime}(0) t, v^{\prime}(0) t\right), \\
& Q(x, y)=\left(Q_{1} x, Q_{2} y\right)=\left((\alpha-1) \int_{0}^{1}(1-s)^{\alpha-2} x(s) d s,(\beta-1) \int_{0}^{1}(1-s)^{\beta-2} y(s) d s\right) .
\end{aligned}
$$

Furthermore, the operator $K_{P}: \operatorname{Im} L \rightarrow \operatorname{dom} L \cap \operatorname{Ker} P$ can be written by

$$
K_{P}(x, y)=\left(I_{0^{+}}^{\alpha} x(t), I_{0^{+}}^{\beta} y(t)\right) .
$$

Proof Obviously, $\operatorname{Im} P=\operatorname{Ker} L$ and $P^{2}(u, v)=P(u, v)$. It follows from $(u, v)=((u, v)-$ $P(u, v))+P(u, v)$ that $\bar{X}=\operatorname{Ker} P+\operatorname{Ker} L$. By simple calculation, we can get that $\operatorname{Ker} L \cap \operatorname{Ker} P=$ $\{(0,0)\}$. Then we get

$$
\bar{X}=\operatorname{Ker} L \oplus \operatorname{Ker} P .
$$

For $(x, y) \in \bar{Y}$, we have

$$
Q^{2}(x, y)=Q\left(Q_{1} x, Q_{2} y\right)=\left(Q_{1}^{2} x, Q_{2}^{2} y\right) .
$$

By the definition of $Q_{1}$, we can get

$$
Q_{1}^{2} x=Q_{1} x \cdot(\alpha-1) \int_{0}^{1}(1-s)^{\alpha-2} d s=Q_{1} x
$$

Similar proof can show that $Q_{2}^{2} y=Q_{2} y$. Thus, we have $Q^{2}(x, y)=Q(x, y)$.

Let $(x, y)=((x, y)-Q(x, y))+Q(x, y)$, where $(x, y)-Q(x, y) \in \operatorname{Ker} Q=\operatorname{Im} L, Q(x, y) \in \operatorname{Im} Q$. It follows from $\operatorname{Ker} Q=\operatorname{Im} L$ and $Q^{2}(x, y)=Q(x, y)$ that $\operatorname{Im} Q \cap \operatorname{Im} L=\{(0,0)\}$. Then, we have

$$
\bar{Y}=\operatorname{Im} L \oplus \operatorname{Im} Q .
$$

Thus

$$
\operatorname{dim} \operatorname{Ker} L=\operatorname{dim} \operatorname{Im} Q=\operatorname{codim} \operatorname{Im} L
$$

This means that $L$ is a Fredholm operator of index zero.

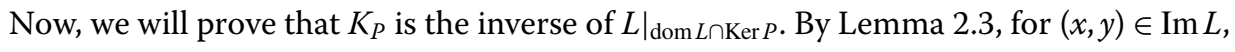
we have

$$
L K_{P}(x, y)=\left(D_{0^{+}}^{\alpha}\left(I_{0^{+}}^{\alpha} x\right), D_{0^{+}}^{\beta}\left(I_{0^{+}}^{\beta} y\right)\right)=(x, y) .
$$


Moreover, for $(u, v) \in \operatorname{dom} L \cap \operatorname{Ker} P$, we have $u^{\prime}(0)=v^{\prime}(0)=0$ and

$$
\begin{aligned}
K_{P} L(u, v) & =\left(I_{0^{+}}^{\alpha} D_{0^{+}}^{\alpha} u(t), I_{0^{+}}^{\beta} D_{0^{+}}^{\beta} v(t)\right) \\
& =\left(u(t)+c_{0}+c_{1} t, v(t)+d_{0}+d_{1} t\right), \quad c_{0}, c_{1}, d_{0}, d_{1} \in \mathbb{R},
\end{aligned}
$$

which, together with $u(0)=v(0)=0$, yields that

$$
K_{P} L(u, v)=(u, v) .
$$

Combining (3.3) with (3.4), we know that $K_{P}=\left(\left.L\right|_{\operatorname{dom} L \cap \operatorname{Ker} P}\right)^{-1}$. The proof is complete.

Lemma 3.3 Assume $\Omega \subset \bar{X}$ is an open bounded subset such that $\operatorname{dom} L \cap \bar{\Omega} \neq \varnothing$, then $N$ is $L$-compact on $\bar{\Omega}$.

Proof By the continuity of $f$ and $g$, we can get that $Q N(\bar{\Omega})$ and $K_{P}(I-Q) N(\bar{\Omega})$ are bounded. So, in view of the Arzelá-Ascoli theorem, we need only prove that $K_{P}(I-Q) N(\bar{\Omega}) \subset \bar{X}$ is equicontinuous.

From the continuity of $f$ and $g$, there exists a constant $A_{i}>0, i=1,2$, such that $\forall(u, v) \in \bar{\Omega}$

$$
\left|\left(I-Q_{1}\right) N_{1} v\right| \leq A_{1}, \quad\left|\left(I-Q_{2}\right) N_{2} u\right| \leq A_{2} .
$$

Furthermore, for $0 \leq t_{1}<t_{2} \leq 1,(u, v) \in \bar{\Omega}$, we have

$$
\begin{aligned}
&\left|K_{P}(I-Q) N\left(u\left(t_{2}\right), v\left(t_{2}\right)\right)-\left(K_{P}(I-Q) N\left(u\left(t_{1}\right), v\left(t_{1}\right)\right)\right)\right| \\
&=\mid\left(I_{0^{+}}^{\alpha}\left(I-Q_{1}\right) N_{1} v\left(t_{2}\right), I_{0^{+}}^{\beta}\left(I-Q_{2}\right) N_{2} u\left(t_{2}\right)\right) \\
& \quad-\left(I_{0^{+}}^{\alpha}\left(I-Q_{1}\right) N_{1} v\left(t_{1}\right), I_{0^{+}}^{\beta}\left(I-Q_{2}\right) N_{2} u\left(t_{1}\right)\right) \mid \\
&=\mid\left(I_{0^{+}}^{\alpha}\left(I-Q_{1}\right) N_{1} v\left(t_{2}\right)-I_{0^{+}}^{\alpha}\left(I-Q_{1}\right) N_{1} v\left(t_{1}\right),\right. \\
&\left.I_{0^{+}}^{\beta}\left(I-Q_{2}\right) N_{2} u\left(t_{2}\right)-I_{0^{+}}^{\beta}\left(I-Q_{2}\right) N_{2} u\left(t_{1}\right)\right) \mid .
\end{aligned}
$$

By

$$
\begin{aligned}
& \left|I_{0^{+}}^{\alpha}\left(I-Q_{1}\right) N_{1} v\left(t_{2}\right)-I_{0^{+}}^{\alpha}\left(I-Q_{1}\right) N_{1} v\left(t_{1}\right)\right| \\
& \quad \leq \frac{1}{\Gamma(\alpha)}\left|\int_{0}^{t_{2}}\left(t_{2}-s\right)^{\alpha-1}\left(I-Q_{1}\right) N_{1} v(s) d s-\int_{0}^{t_{1}}\left(t_{1}-s\right)^{\alpha-1}\left(I-Q_{1}\right) N_{1} v(s) d s\right| \\
& \quad \leq \frac{A_{1}}{\Gamma(\alpha)}\left[\int_{0}^{t_{1}}\left(t_{2}-s\right)^{\alpha-1}-\left(t_{1}-s\right)^{\alpha-1} d s+\int_{t_{1}}^{t_{2}}\left(t_{2}-s\right)^{\alpha-1} d s\right] \\
& \quad=\frac{A_{1}}{\Gamma(\alpha+1)}\left(t_{2}^{\alpha}-t_{1}^{\alpha}\right)
\end{aligned}
$$

and

$$
\begin{aligned}
& \left|\left(I_{0^{+}}^{\alpha}\left(I-Q_{1}\right) N_{1} v\right)^{\prime}\left(t_{2}\right)-\left(I_{0^{+}}^{\alpha}\left(I-Q_{1}\right) N_{1} v\right)^{\prime}\left(t_{1}\right)\right| \\
& \quad=\frac{\alpha-1}{\Gamma(\alpha)}\left|\int_{0}^{t_{2}}\left(t_{2}-s\right)^{\alpha-2}\left(I-Q_{1}\right) N_{1} v(s) d s-\int_{0}^{t_{1}}\left(t_{1}-s\right)^{\alpha-2}\left(I-Q_{1}\right) N_{1} v(s) d s\right|
\end{aligned}
$$




$$
\begin{aligned}
& \leq \frac{A_{1}}{\Gamma(\alpha-1)}\left[\int_{0}^{t_{1}}\left(t_{1}-s\right)^{\alpha-2}-\left(t_{2}-s\right)^{\alpha-2} d s+\int_{t_{1}}^{t_{2}}\left(t_{2}-s\right)^{\alpha-2} d s\right] \\
& \leq \frac{A_{1}}{\Gamma(\alpha)}\left[t_{2}^{\alpha-1}-t_{1}^{\alpha-1}+2\left(t_{2}-t_{1}\right)^{\alpha-1}\right] .
\end{aligned}
$$

Similar proof can show that

$$
\begin{aligned}
& \left|I_{0^{+}}^{\beta}\left(I-Q_{2}\right) N_{2} u\left(t_{2}\right)-I_{0^{+}}^{\beta}\left(I-Q_{2}\right) N_{2} u\left(t_{1}\right)\right| \leq \frac{A_{2}}{\Gamma(\beta+1)}\left(t_{2}^{\beta}-t_{1}^{\beta}\right), \\
& \left|\left(I_{0^{+}}^{\beta}\left(I-Q_{2}\right) N_{2} u\right)^{\prime}\left(t_{2}\right)-\left(I_{0^{+}}^{\beta}\left(I-Q_{2}\right) N_{2} u\right)^{\prime}\left(t_{1}\right)\right| \leq \frac{A_{2}}{\Gamma(\beta)}\left[t_{2}^{\beta-1}-t_{1}^{\beta-1}+2\left(t_{2}-t_{1}\right)^{\beta-1}\right] .
\end{aligned}
$$

Since $t^{\alpha}, t^{\alpha-1}, t^{\beta}$ and $t^{\beta-1}$ are uniformly continuous on $[0,1]$, we can get that $K_{P}(I-$ Q) $N(\bar{\Omega}) \subset \bar{X}$ is equicontinuous.

Thus, we get that $K_{P}(I-Q) N: \bar{\Omega} \rightarrow \bar{X}$ is compact. The proof is complete.

Lemma 3.4 Suppose $\left(H_{1}\right),\left(H_{2}\right)$ hold, then the set

$$
\Omega_{1}=\{(u, v) \in \operatorname{dom} L \backslash \operatorname{Ker} L \mid L(u, v)=\lambda N(u, v), \lambda \in(0,1)\}
$$

is bounded.

Proof Take $(u, v) \in \Omega_{1}$, then $N(u, v) \in \operatorname{Im} L$. By (3.2), we have

$$
\begin{aligned}
& \int_{0}^{1}(1-s)^{\alpha-2} f\left(s, v(s), v^{\prime}(s)\right) d s=0, \\
& \int_{0}^{1}(1-s)^{\beta-2} g\left(s, u(s), u^{\prime}(s)\right) d s=0 .
\end{aligned}
$$

Then, by the integral mean value theorem, there exist constants $\xi, \eta \in(0,1)$ such that $f\left(\xi, v(\xi), v^{\prime}(\xi)\right)=0$ and $g\left(\eta, u(\eta), u^{\prime}(\eta)\right)=0$. So, from $\left(H_{2}\right)$, we get $|v(\xi)| \leq B$ and $|u(\eta)| \leq B$.

From $(u, v) \in \operatorname{dom} L$, we get $u(0)=v(0)=0$, then

$$
\begin{aligned}
& |u(t)|=\left|u(0)+\int_{0}^{t} u^{\prime}(s) d s\right| \leq\left\|u^{\prime}\right\|_{\infty}, \\
& |v(t)|=\left|v(0)+\int_{0}^{t} v^{\prime}(s) d s\right| \leq\left\|v^{\prime}\right\|_{\infty} .
\end{aligned}
$$

By $L(u, v)=\lambda N(u, v)$ and $(u, v) \in \operatorname{dom} L$, we have

$$
u(t)=\frac{\lambda}{\Gamma(\alpha)} \int_{0}^{t}(t-s)^{\alpha-1} f\left(s, v(s), v^{\prime}(s)\right) d s+u^{\prime}(0) t
$$

and

$$
v(t)=\frac{\lambda}{\Gamma(\beta)} \int_{0}^{t}(t-s)^{\beta-1} g\left(s, u(s), u^{\prime}(s)\right) d s+v^{\prime}(0) t .
$$

Then we get

$$
u^{\prime}(t)=\frac{\lambda}{\Gamma(\alpha-1)} \int_{0}^{t}(t-s)^{\alpha-2} f\left(s, v(s), v^{\prime}(s)\right) d s+u^{\prime}(0)
$$


and

$$
v^{\prime}(t)=\frac{\lambda}{\Gamma(\beta-1)} \int_{0}^{t}(t-s)^{\beta-2} g\left(s, u(s), u^{\prime}(s)\right) d s+v^{\prime}(0) .
$$

Take $t=\eta$, we get

$$
u^{\prime}(\eta)=\frac{\lambda}{\Gamma(\alpha-1)} \int_{0}^{\eta}(\eta-s)^{\alpha-2} f\left(s, v(s), v^{\prime}(s)\right) d s+u^{\prime}(0) .
$$

Together with $\left|u^{\prime}(\eta)\right| \leq B,\left(H_{1}\right)$ and (3.6), we have

$$
\begin{aligned}
\left|u^{\prime}(0)\right| & \leq\left|u^{\prime}(\eta)\right|+\frac{\lambda}{\Gamma(\alpha-1)} \int_{0}^{\eta}(\eta-s)^{\alpha-2}\left|f\left(s, v(s), v^{\prime}(s)\right)\right| d s \\
& \leq B+\frac{1}{\Gamma(\alpha-1)} \int_{0}^{\eta}(\eta-s)^{\alpha-2}\left[p_{1}(s)+q_{1}(s)|v(s)|+r_{1}(s)\left|v^{\prime}(s)\right|\right] d s \\
& \leq B+\frac{1}{\Gamma(\alpha-1)} \int_{0}^{\eta}(\eta-s)^{\alpha-2}\left[P_{1}+Q_{1}\|v\|_{\infty}+R_{1}\left\|v^{\prime}\right\|_{\infty}\right] d s \\
& \leq B+\frac{1}{\Gamma(\alpha-1)} \int_{0}^{\eta}(\eta-s)^{\alpha-2}\left[P_{1}+\left(Q_{1}+R_{1}\right)\left\|v^{\prime}\right\|_{\infty}\right] d s \\
& \leq B+\frac{1}{\Gamma(\alpha)}\left[P_{1}+\left(Q_{1}+R_{1}\right)\left\|v^{\prime}\right\|_{\infty}\right] .
\end{aligned}
$$

So, we have

$$
\begin{aligned}
\left\|u^{\prime}\right\|_{\infty} & \leq \frac{1}{\Gamma(\alpha-1)} \int_{0}^{t}(t-s)^{\alpha-2}\left|f\left(s, v(s), v^{\prime}(s)\right)\right| d s+\left|u^{\prime}(0)\right| \\
& \leq \frac{1}{\Gamma(\alpha-1)} \int_{0}^{t}(t-s)^{\alpha-2}\left[p_{1}(s)+q_{1}(s)|v(s)|+r_{1}(s)\left|v^{\prime}(s)\right|\right] d s+\left|u^{\prime}(0)\right| \\
& \leq \frac{1}{\Gamma(\alpha-1)}\left[P_{1}+\left(Q_{1}+R_{1}\right)\left\|v^{\prime}\right\|_{\infty}\right] \int_{0}^{t}(t-s)^{\alpha-2} d s+\left|u^{\prime}(0)\right| \\
& \leq B+\frac{2}{\Gamma(\alpha)}\left[P_{1}+\left(Q_{1}+R_{1}\right)\left\|v^{\prime}\right\|_{\infty}\right] .
\end{aligned}
$$

Similarly, we can get

$$
\left\|v^{\prime}\right\|_{\infty} \leq B+\frac{2}{\Gamma(\beta)}\left[P_{2}+\left(Q_{2}+R_{2}\right)\left\|u^{\prime}\right\|_{\infty}\right]
$$

Together with (3.7) and (3.8), we have

$$
\left\|u^{\prime}\right\|_{\infty} \leq B+\frac{2}{\Gamma(\alpha)}\left\{P_{1}+\left(Q_{1}+R_{1}\right)\left[B+\frac{2}{\Gamma(\beta)}\left(P_{2}+\left(Q_{2}+R_{2}\right)\left\|u^{\prime}\right\|_{\infty}\right)\right]\right\} .
$$

Thus, from $\frac{\Gamma(\alpha) \Gamma(\beta)-4\left(Q_{1}+R_{1}\right)\left(Q_{2}+R_{2}\right)}{\Gamma(\alpha) \Gamma(\beta)}>0$, we obtain that

$$
\left\|u^{\prime}\right\|_{\infty} \leq \frac{\Gamma(\alpha) \Gamma(\beta) B+2 \Gamma(\beta)\left[P_{1}+\left(Q_{1}+R_{1}\right) B\right]+4 P_{2}\left(Q_{1}+R_{1}\right)}{\Gamma(\alpha) \Gamma(\beta)-4\left(Q_{1}+R_{1}\right)\left(Q_{2}+R_{2}\right)}:=M_{1}
$$


and

$$
\left\|v^{\prime}\right\|_{\infty} \leq \frac{1}{\Gamma(\beta)}\left[P_{2}+Q_{2} B+\left(Q_{2}+R_{2}\right) M_{1}\right]:=M_{2} .
$$

Together with (3.5) and (3.6), we get

$$
\|(u, v)\|_{\bar{X}} \leq \max \left\{M_{1}, M_{2}\right\}:=M .
$$

So $\Omega_{1}$ is bounded. The proof is complete.

Lemma 3.5 Suppose $\left(H_{3}\right)$ holds, then the set

$$
\Omega_{2}=\{(u, v) \mid(u, v) \in \operatorname{Ker} L, N(u, v) \in \operatorname{Im} L\}
$$

is bounded.

Proof For $(u, v) \in \Omega_{2}$, we have $(u, v)=\left(c_{1} t, c_{2} t\right), c_{1}, c_{2} \in \mathbb{R}$. Then from $N(u, v) \in \operatorname{Im} L$, we get

$$
\begin{aligned}
& \int_{0}^{1}(1-s)^{\alpha-2} f\left(s, c_{2} s, c_{2}\right) d s=0, \\
& \int_{0}^{1}(1-s)^{\beta-2} g\left(s, c_{1} s, c_{1}\right) d s=0,
\end{aligned}
$$

which, together with $\left(H_{3}\right)$, implies $\left|c_{1}\right|,\left|c_{2}\right| \leq D$. Thus, we have

$$
\|(u, v)\|_{\bar{X}} \leq D
$$

Hence, $\Omega_{2}$ is bounded. The proof is complete.

Lemma 3.6 Suppose the first part of $\left(\mathrm{H}_{3}\right)$ holds, then the set

$$
\Omega_{3}=\{(u, v) \in \operatorname{Ker} L \mid \lambda(u, v)+(1-\lambda) Q N(u, v)=(0,0), \lambda \in[0,1]\}
$$

is bounded.

Proof For $(u, v) \in \Omega_{3}$, we have $(u, v)=\left(c_{1} t, c_{2} t\right), c_{1}, c_{2} \in \mathbb{R}$ and

$$
\begin{aligned}
& \lambda c_{1} t+(1-\lambda)(\alpha-1) \int_{0}^{1}(1-s)^{\alpha-2} f\left(s, c_{2} s, c_{2}\right) d s=0, \\
& \lambda c_{2} t+(1-\lambda)(\beta-1) \int_{0}^{1}(1-s)^{\beta-2} g\left(s, c_{1} s, c_{1}\right) d s=0 .
\end{aligned}
$$

If $\lambda=0$, then $\left|c_{1}\right|,\left|c_{2}\right| \leq D$ because of the first part of $\left(H_{3}\right)$. If $\lambda=1$, then $c_{1}=c_{2}=0$. For $\lambda \in(0,1]$, we can obtain $\left|c_{1}\right|,\left|c_{2}\right| \leq D$. Otherwise, if $\left|c_{1}\right|$ or $\left|c_{2}\right|>D$, in view of the first part of $\left(H_{3}\right)$, one has

$$
\lambda c_{1}^{2} t+(1-\lambda)(\alpha-1) \int_{0}^{1}(1-s)^{\alpha-2} c_{1} f\left(s, c_{2} s, c_{2}\right) d s>0,
$$


or

$$
\lambda c_{2}^{2} t+(1-\lambda)(\beta-1) \int_{0}^{1}(1-s)^{\beta-2} c_{2} g\left(s, c_{1} s, c_{1}\right) d s>0,
$$

which contradicts (3.9) or (3.10). Therefore, $\Omega_{3}$ is bounded. The proof is complete.

Remark 3.1 If the second part of $\left(H_{3}\right)$ holds, then the set

$$
\Omega_{3}^{\prime}=\{(u, v) \in \operatorname{Ker} L \mid-\lambda(u, v)+(1-\lambda) Q N(u, v)=(0,0), \lambda \in[0,1]\}
$$

is bounded.

Proof of Theorem 3.1 Set $\Omega=\left\{(u, v) \in \bar{X} \mid\|(u, v)\|_{\bar{X}}<\max \{M, D\}+1\right\}$. It follows from Lemma 3.2 and 3.3 that $L$ is a Fredholm operator of index zero and $N$ is $L$-compact on $\bar{\Omega}$. By Lemma 3.4 and 3.5, we get that the following two conditions are satisfied:

(1) $L(u, v) \neq \lambda N(u, v)$ for every $((u, v), \lambda) \in[(\operatorname{dom} L \backslash \operatorname{Ker} L) \cap \partial \Omega] \times(0,1)$;

(2) $N x \notin \operatorname{Im} L$ for every $(u, v) \in \operatorname{Ker} L \cap \partial \Omega$.

Take

$$
H((u, v), \lambda)= \pm \lambda(u, v)+(1-\lambda) Q N(u, v)
$$

According to Lemma 3.6 (or Remark 3.1), we know that $H((u, v), \lambda) \neq 0$ for $(u, v) \in \operatorname{Ker} L \cap$ $\partial \Omega$. Therefore,

$$
\begin{aligned}
\operatorname{deg}\left(\left.Q N\right|_{\operatorname{Ker} L}, \Omega \cap \operatorname{Ker} L,(0,0)\right) & =\operatorname{deg}(H(\cdot, 0), \Omega \cap \operatorname{Ker} L,(0,0)) \\
& =\operatorname{deg}(H(\cdot, 1), \Omega \cap \operatorname{Ker} L,(0,0)) \\
& =\operatorname{deg}( \pm I, \Omega \cap \operatorname{Ker} L,(0,0)) \neq 0 .
\end{aligned}
$$

So, the condition (3) of Lemma 2.1 is satisfied. By Lemma 2.1, we can get that $L(u, v)=$ $N(u, v)$ has at least one solution in $\operatorname{dom} L \cap \bar{\Omega}$. Therefore, BVP $(1.1)$ has at least one solution. The proof is complete.

\section{Example}

Example 4.1 Consider the following BVP:

$$
\left\{\begin{array}{l}
D_{0^{+}}^{\frac{3}{2}} u(t)=\frac{1}{16}[v(t)-10]+\frac{t^{2}}{16} e^{-\left|v^{\prime}(t)\right|}, \quad t \in[0,1], \\
D_{0^{+}}^{\frac{5}{4}} v(t)=\frac{1}{12}[u(t)-8]+\frac{t^{3}}{12} \sin ^{2}\left(u^{\prime}(t)\right), \quad t \in[0,1], \\
u(0)=v(0)=0, \quad u^{\prime}(0)=u^{\prime}(1), \quad v^{\prime}(0)=v^{\prime}(1) .
\end{array}\right.
$$

Choose $p_{1}(t)=\frac{11}{16}, p_{2}(t)=\frac{3}{4}, q_{1}(t)=\frac{1}{16}, q_{2}(t)=\frac{1}{12}, r_{1}(t)=r_{2}(t)=0, B=D=10$.

By simple calculation, we can get that $\left(H_{1}\right),\left(H_{2}\right)$ and the first part of $\left(H_{3}\right)$ hold.

By Theorem 3.1, we obtain that BVP (4.1) has at least one solution. 
Authors' contributions

All authors typed, read and approved the final manuscript.

\section{Acknowledgements}

The authors would like to thank the referees very much for their helpful comments and suggestions. This research was supported by the Fundamental Research Funds for the Central Universities (2010LKSX09) and the National Natural Science Foundation of China (11271364).

\section{Received: 14 May 2012 Accepted: 22 August 2012 Published: 7 September 2012}

\section{References}

1. Metzler, R, Klafter, J: Boundary value problems for fractional diffusion equations. Physica A 278, 107-125 (2000)

2. Scher, H, Montroll, E: Anomalous transit-time dispersion in amorphous solids. Phys. Rev. B 12, 2455-2477 (1975)

3. Mainardi, F: Fractional diffusive waves in viscoelastic solids. In: Wegner, JL, Norwood, FR (eds.) Nonlinear Waves in Solids, pp. 93-97. ASME/AMR, Fairfield (1995)

4. Diethelm, K, Freed, AD: On the solution of nonlinear fractional order differential equations used in the modeling of viscoplasticity. In: Keil, F, Mackens, W, Voss, H, Werther, J (eds.) Scientific Computing in Chemical Engineering II-Computational Fluid Dynamics, Reaction Engineering and Molecular Properties, pp. 217-224. Springer, Heidelberg (1999)

5. Gaul, L, Klein, P, Kempfle, S: Damping description involving fractional operators. Mech. Syst. Signal Process. 5, 81-88 (1991)

6. Glockle, WG, Nonnenmacher, TF: A fractional calculus approach of self-similar protein dynamics. Biophys. J. 68, 46-53 (1995)

7. Mainardi, F: Fractional calculus: some basic problems in continuum and statistical mechanics. In: Carpinteri, A, Mainardi, F (eds.) Fractals and Fractional Calculus in Continuum Mechanics, pp. 291-348. Springer, Vienna (1997)

8. Metzler, F, Schick, W, Kilian, HG, Nonnenmacher, TF: Relaxation in filled polymers: a fractional calculus approach. J. Chem. Phys. 103, 7180-7186 (1995)

9. Oldham, KB, Spanier, J: The Fractional Calculus. Academic Press, New York (1974)

10. Agarwal, RP, O'Regan, D, Stanek, S: Positive solutions for Dirichlet problems of singular nonlinear fractional differential equations. J. Math. Anal. Appl. 371, 57-68 (2010)

11. Bai, Z, Hu, L: Positive solutions for boundary value problem of nonlinear fractional differential equation. J. Math. Anal. Appl. 311, 495-505 (2005)

12. Kaufmann, ER, Mboumi, E: Positive solutions of a boundary value problem for a nonlinear fractional differential equation. Electron. J. Qual. Theory Differ. Equ. 3, 1-11 (2008)

13. Jafari, H, Gejji, VD: Positive solutions of nonlinear fractional boundary value problems using Adomian decomposition method. Appl. Math. Comput. 180, 700-706 (2006)

14. Benchohra, M, Hamani, S, Ntouyas, SK: Boundary value problems for differential equations with fractional order and nonlocal conditions. Nonlinear Anal. 71, 2391-2396 (2009)

15. Liang, S, Zhang, J: Positive solutions for boundary value problems of nonlinear fractional differential equation. Nonlinear Anal. 71, 5545-5550 (2009)

16. Zhang, S: Positive solutions for boundary-value problems of nonlinear fractional differential equations. Electron. J. Differ. Equ. 36, 1-12 (2006)

17. Kosmatov, N: A boundary value problem of fractional order at resonance. Electron. J. Differ. Equ. 135, 1-10 (2010)

18. Wei, Z, Dong, W, Che, J: Periodic boundary value problems for fractional differential equations involving a Riemann-Liouville fractional derivative. Nonlinear Anal. 73, 3232-3238 (2010)

19. Bai, Z, Zhang, Y: Solvability of fractional three-point boundary value problems with nonlinear growth. Appl. Math. Comput. 218(5), 1719-1725 (2011)

20. Bai, Z: Solvability for a class of fractional m-point boundary value problem at resonance. Comput. Math. Appl. 62(3), 1292-1302 (2011)

21. Ahmad, B, Sivasundaram, S: On four-point nonlocal boundary value problems of nonlinear integrodifferential equations of fractional order. Appl. Math. Comput. 217, 480-487 (2010)

22. Wang, G, Ahmad, B, Zhang, L: Impulsive anti-periodic boundary value problem for nonlinear differential equations of fractional order. Nonlinear Anal. 74, 792-804 (2011)

23. Yang, L, Chen, H: Unique positive solutions for fractional differential equation boundary value problems. Appl. Math. Lett. 23, 1095-1098 (2010)

24. Yang, L, Chen, H: Nonlocal boundary value problem for impulsive differential equations of fractional order. Adv. Differ. Equ. 2011, 1-16 (2011)

25. Jiang, W: The existence of solutions to boundary value problems of fractional differential equations at resonance. Nonlinear Anal. 74, 1987-1994 (2011)

26. Su, X: Boundary value problem for a coupled system of nonlinear fractional differential equations. Appl. Math. Lett. 22, 64-69 (2009)

27. Bai, C, Fang, J: The existence of a positive solution for a singular coupled system of nonlinear fractional differential equations. Appl. Math. Comput. 150, 611-621 (2004)

28. Ahmad, B, Alsaedi, A: Existence and uniqueness of solutions for coupled systems of higher-order nonlinear fractional differential equations. Fixed Point Theory Appl. 2010, 1-17 (2010)

29. Ahmad, B, Nieto, J: Existence results for a coupled system of nonlinear fractional differential equations with three-point boundary conditions. Comput. Math. Appl. 58, 1838-1843 (2009)

30. Rehman, M, Khan, R: A note on boundary value problems for a coupled system of fractional differential equations. Comput. Math. Appl. 61, 2630-2637 (2011)

31. Su, X: Existence of solution of boundary value problem for coupled system of fractional differential equations. Eng. Math. 26, 134-137 (2009)

32. Yang, W: Positive solutions for a coupled system of nonlinear fractional differential equations with integral boundary conditions. Comput. Math. Appl. 63, 288-297 (2012) 
33. Zhang, Y, Bai, Z, Feng, T: Existence results for a coupled system of nonlinear fractional three-point boundary value problems at resonance. Comput. Math. Appl. 61(4), 1032-1047 (2011)

34. Mawhin, J: Topological degree and boundary value problems for nonlinear differential equations in topological methods for ordinary differential equations. Lect. Notes Math. 1537, 74-142 (1993)

35. Lakshmikantham, V, Leela, S, Vasundhara Devi, J: Theory of Fractional Dynamic Systems. Cambridge Academic Publishers, Cambridge (2009)

doi:10.1186/1687-2770-2012-98

Cite this article as: Hu et al.: Existence of solutions for a coupled system of fractional differential equations at resonance. Boundary Value Problems 2012 2012:98.

Submit your manuscript to a SpringerOpen ${ }^{\circ}$ journal and benefit from:

- Convenient online submission

- Rigorous peer review

Immediate publication on acceptance

- Open access: articles freely available online

- High visibility within the field

- Retaining the copyright to your article 